\title{
Composition en acides gras libres et en acides aminés de deux fromages fabriqués à partir de lait de chèvre
}

\author{
M. BALTADJIEVA*, G. KALATZOPOULOS**, V. STAMENOVA* \\ et A. SFAKIANOS**
}

\section{RESUME}

Sur une série de préparations de deux nouveaux types de fromages fabriqués à partir de lait de chèvre selon des technologies standardisées, nous avons suivi pendant l'affinage l'évolution de la protéolyse ainsi que les compositions en acides aminés et en acides gras libres volatils (A.G.L.V.).

Le premier fromage, le Rumelia, de type « pasta filata ", préparé avec un levain de bactéries lactiques thermophiles, présente un degré de maturation déjà assez avancé à partir du $2^{\mathrm{e}}$ mois. Les acides aminés libres dominants sont l'acide glutamique, la lysine, la valine, la leucine et l'isoleucine. Les A.G.L.V., en quantité importante, lui donnent un goût trop piquant mais la présence des acides caproïque et caprylique lui confère l'arôme caractéristique des tromages de chèvre.

Le second fromage, de type semi-dur, proche de l'Edam, est préparé avec des ferments lactiques mésophiles. Son degré de maturation est un peu moins poussé que celui du précédent et la moindre libération des A.G.L.V. lui donne un goût moins piquant et plus doux.

Mots clés : Fromages de lait de chèvre - Type Pasta filata - Type Edam Affinage - Protéolyse - Lipolyse.

* Institut de Technologie alimentaire de Plovdiv (Bulgarie).

** Ecole Supérieure d'agriculture d'Athènes, Votanikos (Grèce). 


\section{INTRODUCTION}

Dans le cadre d'un programme commun entre les deux laboratoires homologues de Grèce et de Bulgarie, pour une meilleure valorisation des laits de brebis et de chèvre produits en abondance dans les deux pays, nous avons mis au point quatre types de fabrications de fromages de chèvre : un type semi-dur comparable à l'Edam, un type "pasta filata " nommé Rumelia, un type Sainte Maure et un type Kefalotyri nommé Fildrus (Baltadjieva et al., 1978; Kalatzopoulos et al., 1983; Veinoglou et al., 1983; Baltadjieva et al., 1984).

Les fromages obtenus étaient de bonne qualité et bien acceptés par les consommateurs.

Pour les fromages semi-durs type Edam et pour le Rumelia, nous avons cherché à préciser l'évolution de leur composition au cours de l'affinage, notamment l'importance de la dégradation des protéines et de la libération des acides gras volatils.

Cette étude représente une première approche visant à caractériser la maturation de fromages faits à partir du lait de chèvre. Certes, le phénomène biochimique de la maturation a fait l'objet de nombreux travaux et pour les grands types de fromages au lait de vache on dispose de données relatives à l'évolution de leur composition au cours de l'affinage (Lenoir, 1962, 1963; Do Ngoc, Lenoir et Choisy, 1971 ; Kuzdzal-Savoie et al., 1971; Rasic, Vucurovic et Obradovic, 1973; Harper, Kristoffersen et Wang, 1978 ; Polychroniadou et Vlachos, 1979; Aston et Dulley, 1982; Kasprzyk et al., 1983 ; Choisy et al., 1984) mais, à notre connaissance, il n'existe pas d'étude portant sur la maturation des fromages de chèvre.

On connaît la complexité du phénomène d'affinage, la grande variété des agents responsables, microbes et enzymes, la diversité des modifications subies par les constituants et par suite le très grand nombre de produits formés. Dans une première étape, nous avons choisi d'étudier plus particulièrement la libération des acides aminés et des acides gras libres volatils dont la contribution au développement de la saveur et de l'arôme peut être décisive.

Compte tenu de l'importance des fromages faits à partir du lait de chèvre nous pensons, dans un proche avenir, pouvoir compléter cette étude par celle d'autres composés.

\section{MATERIEL ET METHODES}

\section{A. Fromage Rumelia}

Une série d'essais conduits en Bulgarie et en Grèce, a permis la mise au point d'une technologie ayant fait l'objet d'un brevet du Gouvernement bulgare ( $\left.N^{\circ} 31963 / 1982\right)$ (Baltadjieva et al., 1984). 
TABLEAU $1-T A B L E 1$

Composition du fromage Rumelia - Composition of Rumelia cheese

\begin{tabular}{|c|c|c|c|c|c|}
\hline & 10 jours & 30 jours & 60 jours & 90 jours & 120 jours \\
\hline $\begin{array}{c}\text { Humidité \%* } \\
\text { (Min.- Max.) }\end{array}$ & $\begin{array}{c}43,2 \\
(41,3-44,5)\end{array}$ & $\begin{array}{c}41,6 \\
(39,8-42,6)\end{array}$ & $\begin{array}{c}40,6 \\
(39,6-41,7)\end{array}$ & $\begin{array}{c}39,4 \\
(39,0-41,0)\end{array}$ & $\begin{array}{c}39,0 \\
(38,9-40,1)\end{array}$ \\
\hline $\begin{array}{c}\text { Matière grasse \%* } \\
\text { (Min.-!̣ax.) }\end{array}$ & $\begin{array}{c}27,5 \\
(25,3-28,6)\end{array}$ & $\begin{array}{c}28,6 \\
(26,2-30,2)\end{array}$ & $(26,8-30,7)$ & $(27,2-30,9)$ & $(28,6-31,2)$ \\
\hline $\begin{array}{c}\text { Mat.gras/Mat. } \\
\text { sèche \% * }\end{array}$ & 48,3 & 49,0 & 49,2 & 48,7 & 49,2 \\
\hline $\begin{array}{c}\text { Acidité en } \\
\text { Acide lactique \%* }\end{array}$ & 1,62 & 1,81 & 1,77 & 1,50 & 1,42 \\
\hline Naci \%* & $2, ? 7$ & 2,55 & 2,75 & 3,84 & 2,98 \\
\hline
\end{tabular}

* Valeurs moyennes à divers temps de la maturation. 
Les échantillons examinés relèvent d'une série de dix préparations réalisées en Bulgarie et en Grèce dans les mêmes conditions. Les levains utilisés étaient constitués par l'association Lactobacillus bulgaricus et Streptococcus thermophilus dans un rapport 1/3.

\section{B. Fromage type Edam}

Le fromage a été préparé à partir du lait de chèvre, selon la technologie de l'Edam adaptée à ce lait (Kalatzopoulos et al., 1983), le but étant d'obtenir un fromage à pâte semi-dure à goût piquant. Les échantillons examinés appartiennent à une série de neuf préparations, faites dans chaque pays selon un protocole standardisé. Les levains utilisés étaient constitués d'espèces mésophiles : Streptococcus lactis, $S$. cremoris et $S$. diacetylactis.

\section{Méthodes d'analyses}

Les acides gras libres volatils ont été extraits et dosés selon la méthode de Dimitrov, Obretenova et Obretenov (1976). Les acides sont collectés par double distillation après alcalinisation puis acidification de l'échantillon. Les deux distillats, saturés par le chlorure de sodium, sont extraits par l'éther éthylique. Les acides gras volatils sont analysés par chromatographie en phase gazeuse, dans les conditions suivantes : colonne avec support solide de chromosorb W (60-80 mesh) imprégné de $10 \%$ de Carbowax 20 additionné d'acide terephtalique; gaz vecteur : azote $(45 \mathrm{ml} / \mathrm{min})$; température réglée en isotherme à $156^{\circ} \mathrm{C}$.

Les acides aminés ont été dosés par la méthode de Inihof et Brio (1971), le fractionnement étant fait sur une colonne Dowex-50 avec un appareil type HD-1200 E.

Le degré de protéolyse a été déterminé par précipitation fractionnée et dosage de l'azote des fractions par la méthode Kjeldhal, l'azote peptonique étant défini comme la fraction soluble en solution alcaline $(\mathrm{NaCl})$ précipitable par le tanin.

Les dosages de l'humidité, de la matière grasse et du chlorure de sodium ont été réalisés selon les méthodes normalisées de la F.I.L.

\section{RESULTATS}

Les fromages examinés étaient de bonne qualité, homogènes, sans défauts de croûte ou de pâte, de goût agréable, piquant, avec l'arôme spécifique, mais non intense, des fromages de chèvre. 


\section{A. Fromage Rumelia}

La composition du fromage, pendant la maturation, est présentée sur le tableau 1. Le tableau 2 rend compte de l'évolution des différentes fractions azotées. Le tableau 3 présente les teneurs en acides aminés libres et le tableau 4 celles en acides gras libres volatils à divers stades de l'affinage.

Ces résultats concernent les fromages fabriqués en Bulgarie. Parallèlement, des fromages fabriqués en Grèce, selon la même technologie, ont été examinés; les résultats correspondants seront analysés au cours de la discussion.

\section{B. Fromage semi-dur type Edam}

Dans le même ordre que celui adopté dans le paragraphe précédent, le tableau 5 présente la composition du fromage, le tableau 6 l'évolution des différentes fractions azotées, le tableau 7 les teneurs en acides aminés libres et le tableau 8 celles en acides gras libres volatils.

\section{DISCUSSION}

\section{A. Fromage Rumelia}

1. Composition chimique, degré de protéolyse et composition en acides aminés

Les fromages préparés présentent des caractéristiques de composition en matière sèche et matière grasse qui, selon les normes grecques, peuvent être considérées comme normales et les teneurs en sel se situent dans les limites désirées par les consommateurs des Balkans.

L'acidité augmente jusqu'au $2^{\mathrm{e}}$ mois, ensuite elle diminue. Malgré une acidité relativement élevée, le fromage n'avait pas un goût acide mais le goût piquant était prédominant.

L'examen du tableau 2 permet de relever l'allure de la protéolyse. Le coefficient de maturation, défini par le rapport $\mathrm{N}$ soluble/ $\mathrm{N}$ total, augmente assez fortement jusqu'au $2^{\mathrm{e}}$ mois, il tend ensuite à se stabiliser à une valeur proche de celles qui ont été observées sur d'autres types de fromages (Lenoir, 1962, 1963). $\mathrm{La}$ teneur en azote peptonique s'accroît également jusqu'au $2^{\mathrm{e}}$ mois, tandis que celle en azote aminé continue à augmenter jusqu'au $4^{\mathrm{e}}$ mois, et elle atteint en fin de maturation $13 \%$ de l'azote total, soit $38 \%$ de l'azote soluble. Ces valeurs s'accordent avec les teneurs élevées en acides aminés libres, $3,35 \mathrm{~g}$ pour $100 \mathrm{~g}$ de fromage (tab. 3), un niveau comparable à celui observé sur Gruyère de Comté, dont les taux se situent de 2,4 à 4,2 g (Do Ngoc, Lenoir et Choisy, 1971). 


\section{$T A B L E A U 2-T A B L E 2$}

Evolution des fractions azotées du fromage Rumelia au cours de la maturation Evolution in nitrogen fraction of the Rumelia cheese during the ripening

\begin{tabular}{|c|c|c|c|c|c|}
\hline Fractions azotées* & 10 jours & 30 jours & 60 jours & 90 jours & 120 jours \\
\hline $\begin{array}{c}\text { Azote total (NT) } \\
\%\end{array}$ & 3,87 & 3,95 & 4,13 & 4,22 & 4,26 \\
\hline $\begin{array}{c}\text { Azote soluble } \\
(\% \text { NT) }\end{array}$ & $1,6,9$ & 21,5 & 31,0 & 32,3 & 34,5 \\
\hline $\begin{array}{c}\text { Azote peptonique } \\
(\% \text { NT) }\end{array}$ & 13,5 & 14,7 & 20,8 & 21,1 & 11,2 \\
\hline $\begin{array}{c}\text { Azote aminé } * * \\
(\% \text { NT) }\end{array}$ & 3,4 & 6,7 & 10,2 & 32,8 & 34,6 \\
\hline $\begin{array}{c}\text { Azote aminé } * * \\
(\% \text { N soluble) }\end{array}$ & 20,1 & 31,2 & 38,1 \\
\hline
\end{tabular}

* Valeurs moyennes à divers temps de la maturation.

** Azote des acides aminés libres + azote ammoniacal 
La figure 1 concrétise les évolutions de la teneur en azote soluble et en acides aminés libres au cours de la maturation.

Les données du tableau 3 et de la figure 2 mettent en évidence la présence dominante de certains acides aminés, notamment celle de l'acide glutamique, de la lysine et de la leucine qui, ensemble en fin d'affinage, constituent environ $45 \%$ des amino-acides totaux. En comparant l'histogramme de la figure 2 avec celui établi par Do Ngoc, Lenoir et Choisy (1971) sur des fromages de Gruyère de Comté affinés on peut observer une certaine similitude, surtout pour ce qui concerne les acides aminés prédominants, tandis que des différences notables apparaissent avec, par exemple, le profil chromatographique caractéristique du Saint-Paulin.

Il est à remarquer que les pourcentages des amino-acides du fromage le $1^{\text {er }}$ mois peuvent être sensiblement différents de ceux relevés le $4^{\mathrm{e}}$ mois. Ainsi, les proportions relatives d'acide glutamique, d'alanine, de valine, d'isoleucine augmentent au cours de l'affinage; en revanche, celles de thréonine, de sérine, de proline et de tyrosine diminuent. Ces différences peuvent être dues aux dégradations secondaires des acides aminés, comme par exemple la décarboxylation dans le cas de la tyrosine ou la désamination dans celui

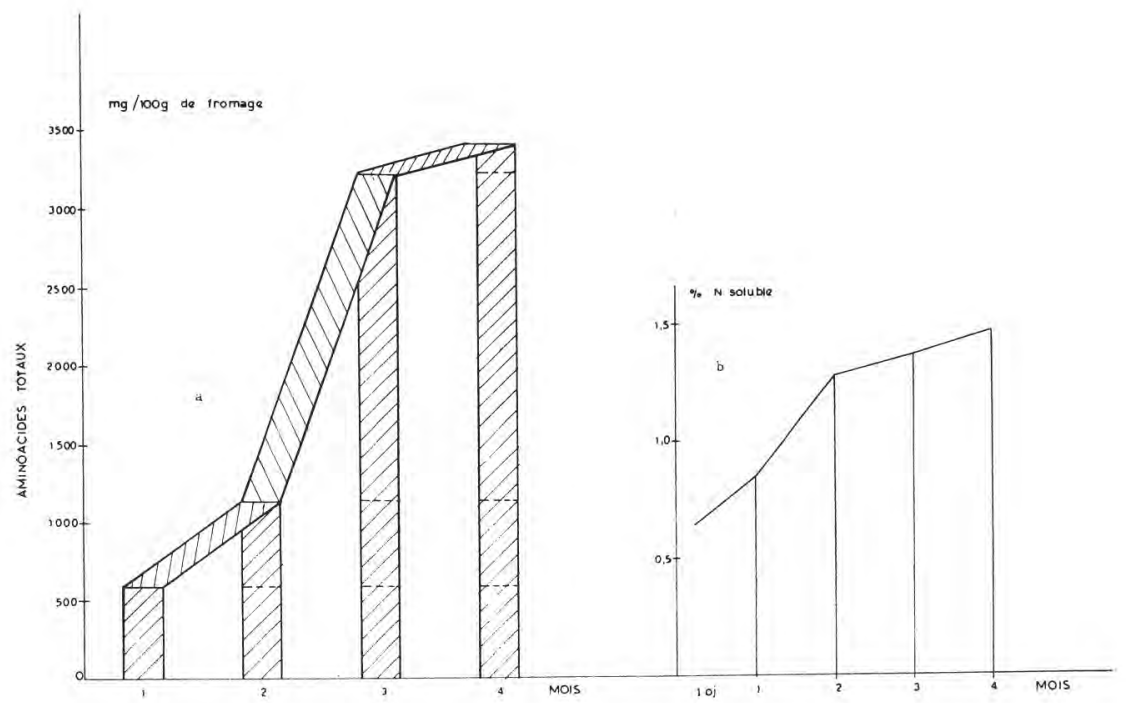

fig. 1

Evolution des teneurs en acides aminés libres (a) et en azote soluble (b) au cours de l'affinage du fromage Rumelia.

Evolution of free aminoacids content (a) and N.P.N. content (b) during Rumelia cheese ripening. 
$T A B L E A U 3-T A B L E 3$

Composition en acides aminés libres du fromage Rumelia (en $\mathrm{mg} / 100 \mathrm{~g}$ de fromage)

Composition in free aminoacids of Rumelia cheese (in $\mathrm{mg} / 100 \mathrm{~g}$ of cheese)

\begin{tabular}{|c|c|c|c|c|c|c|c|c|}
\hline Acides aminés * & 1mois & $q$ & 2 mois & $q$ & 3 mois & $\%$ & 4 mois & $\%$ \\
\hline Lysine & $68,49 \pm 4,30$ & 11,60 & $161,96 \pm 16,77$ & 14,2 & $383,85 \pm 23,92$ & 12,13 & $39,21 \pm 19,80$ & 11,60 \\
\hline Histidine & $14,79 \pm 2,58$ & 2,50 & $28,52 \pm 3,78$ & 2,51 & $82,40 \pm 5,17$ & 2,60 & $86,35 \pm 5,20$ & 2,60 \\
\hline Arginine & $1,61 \pm 0,49$ & 0,27 & $5,9 \underline{ \pm} \pm 1,71$ & 0,52 & $23,63 \pm 3,16$ & 0,75 & $25,44 \pm 2,14$ & 0,76 \\
\hline Aspartique (acide) & $10,05 \pm 1,23$ & 1,70 & $20,51 \pm 1,88$ & 1,80 & $52,73 \pm 3,95$ & 1,67 & $53,08 \pm 2,43$ & 1,58 \\
\hline Thréonine & $63,4 \pm 6,31$ & 10,77 & $119,11 \pm 7,84$ & 10,47 & $159,83 \pm 9,15$ & 5,05 & $164,24 \pm 8,22$ & 4,89 \\
\hline Serine & $49,05 \pm 4,64$ & 8,30 & $91,20 \pm 6,12$ & 8,00 & $215,02 \pm 14,45$ & 6,80 & $214,63 \pm 9,08$ & 6,40 \\
\hline Glutamique (acide) & $91,34 \pm 9,76$ & 15,50 & $159,84 \pm 22,57$ & 14,05 & $655,01 \pm 49,54$ & 20,70 & $713,22 \pm 33,97$ & 21,23 \\
\hline Proline & $50,50 \pm 5,68$ & 8,57 & $122,15 \pm 11,44$ & 10,74 & $176,03 \pm 6,61$ & 5,56 & $189,78 \pm 6,73$ & 5,65 \\
\hline Glycine & $29,17 \pm 3,81$ & 4,95 & $35,59 \pm 3,98$ & 3,13 & $110,69 \pm 10,48$ & 3,50 & $127,78 \pm 15,93$ & 3,80 \\
\hline Alanine & $14,02 \pm 1,44$ & 2,38 & $30,99 \pm 1,50$ & 2,72 & $105,47 \pm 5,61$ & 3,30 & $122,19 \pm 4,27$ & 3,64 \\
\hline Valine & $39,90 \pm 3,60$ & 6,77 & $75,40 \pm 5,58$ & 6,63 & $263,31 \pm 13,80$ & 8,32 & $307,42 \pm 27,18$ & 9,15 \\
\hline Methionine & $14,37 \pm 1,45$ & 2,44 & $21,51 \pm 2,51$ & 1,90 & $111,94 \pm 8,21$ & 3,54 & $116,70 \pm 5,26$ & 3,47 \\
\hline Isoleucine & $27,15 \pm 2,59$ & 4,61 & $55,69 \pm 3,04$ & 4,90 & $203,22 \pm 10,11$ & 6,42 & $207,70 \pm 7,37$ & 6,18 \\
\hline Leucine & $69,23 \pm 3,27$ & 11,75 & $108,99 \pm 4,58$ & 9,60 & $389,14 \pm 15,40$ & 12,29 & $401,47 \pm 2,45$ & 11,95 \\
\hline Tyrosine & $12,90 \pm 1,38$ & 2,19 & $28,66 \pm 1,99$ & 2,50 & $49,51 \pm 2,30$ & 1,56 & $52,10 \pm 14,99$ & 1,55 \\
\hline Phénylalanine & $33,73 \pm 1,72$ & 5,73 & $71,49 \pm 6,20$ & 6,28 & $183,80 \pm 6,28$ & 5,81 & $185,06 \pm$ & 5,51 \\
\hline Acides aminés totaux & 589,68 & 100 & 1137,55 & 100 & 3165,56 & 100 & 3359,37 & 100 \\
\hline
\end{tabular}

* Valeurs moyennes à divers temps de la maturation, 


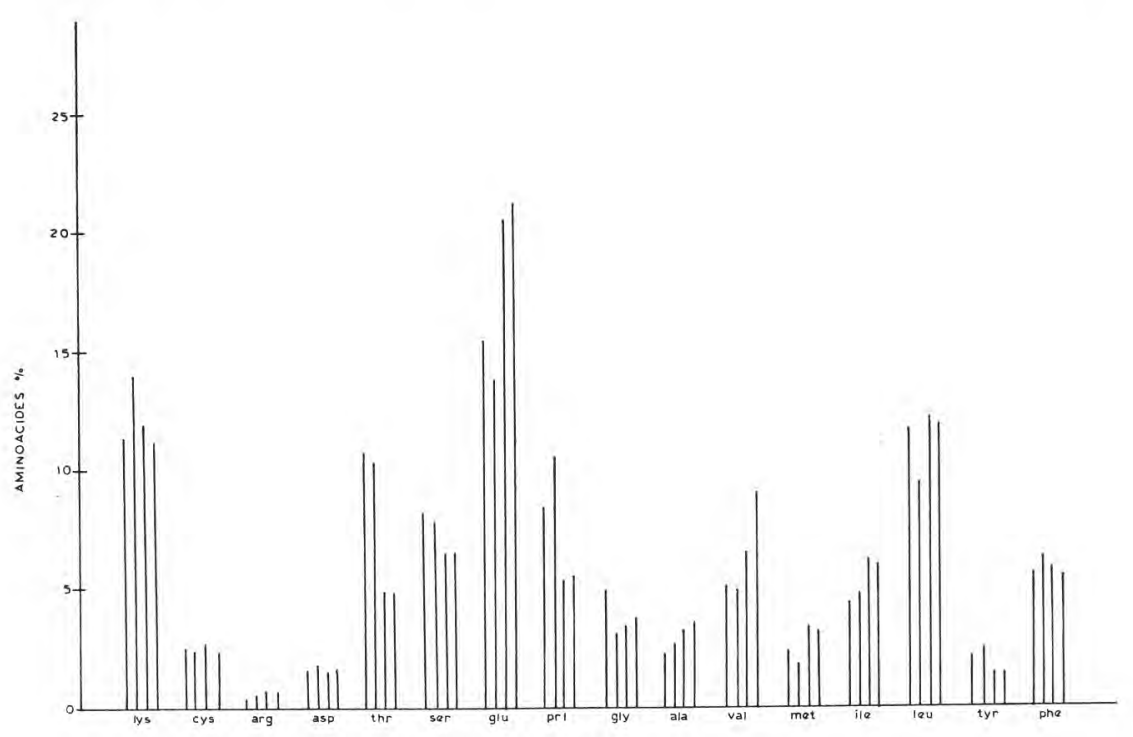

fig. 2

Variations de la composition en acides aminés libres du fromage Rumelia au cours de l'affinage. (Proportions exprimées en pour cent des acides aminés totaux).

Variations of free aminoacids content of Rumelia cheese during ripening. (Proportions expressed in per cent of total aminoacids).

de la sérine. Elles peuvent aussi s'expliquer par une libération préférentielle lors de la protéolyse, par une production à partir des micro-organismes, excrétion ou lyse microbienne, ou par des réactions de transamination.

La composition en acides aminés des fromages fabriqués en Grèce, selon la même technologie, est comparable à celle des fromages bulgares. Les seules différences, d'ailleurs non significatives, concernent les pourcentages d'arginine et de thréonine.

\section{Composition en acides gras libres volatils}

Il a été noté précédemment que les fromages avaient un goût assez piquant; ce caractère est dû à la présence de quantités importantes d'acides gras libres volatils comme on peut le constater à l'examen du tableau 4. Les origines de ces acides peuvent être diverses. Les acides acétique et propionique résultent principalement de fermentations des hydrates de carbone ou des lactates mais ils peuvent aussi provenir de la transformation des acides aminés. Les acides isobutyrique et isovalérique ont pour origine prédominante le catabolisme de certains acides aminés qui sont 
TABLEAU $4-T A B L E 4$

Composition en acides gras libres volatils du fromage Rumelia

Composition in volatile free fatty acids of Rumelia cheese

\begin{tabular}{|c|c|c|c|c|c|c|c|c|c|}
\hline \multirow{2}{*}{\multicolumn{2}{|c|}{ Acides gras }} & \multicolumn{2}{|c|}{ 1er mois } & \multicolumn{2}{|c|}{ 2ême mois } & \multicolumn{2}{|c|}{ 3ême mois } & \multicolumn{2}{|c|}{$4^{\text {ëme }}$ mois } \\
\hline & & $\mathrm{g} / \mathrm{Kg}$ From. & $\%$ & g/Kg From. & $\%$ & g/Kg From. & $\%$ & $\mathrm{~g} / \mathrm{Kg}$ From. & $\%$ \\
\hline Propionique & $\begin{array}{l}\text { Moyenne } \\
\text { Min.Max. }\end{array}$ & $\begin{array}{c}0,168 \\
0,15-0,18\end{array}$ & 5,7 & $\begin{array}{c}0,164 \\
0,16-0,18\end{array}$ & 4,0 & $\begin{array}{c}0,145 \\
0,14-0,15\end{array}$ & 3,2 & $\begin{array}{c}0,110 \\
0,09-0,12\end{array}$ & 2,2 \\
\hline Isobutyrique & $\begin{array}{l}\text { Moyenne } \\
\text { Min.Max. }\end{array}$ & $\begin{array}{c}0,265 \\
0,25-0,28\end{array}$ & 9,0 & $\begin{array}{c}0,311 \\
0,30-0,33\end{array}$ & 7,6 & $\begin{array}{c}0,181 \\
0,17-0,19\end{array}$ & 4,0 & $\begin{array}{c}0,150 \\
0,13-0,16\end{array}$ & 3,0 \\
\hline Butyrique & $\begin{array}{l}\text { Moyenne } \\
\text { Min.Max. }\end{array}$ & $\begin{array}{c}0,618 \\
0,59-0,64\end{array}$ & 21,0 & $\begin{array}{c}0,973 \\
0,89-0,99\end{array}$ & 23,8 & $\begin{array}{c}1,147 \\
1,09-1,21\end{array}$ & 25,4 & $\begin{array}{c}1,342 \\
1,27-1,45\end{array}$ & 26,9 \\
\hline Isovalêrique & $\begin{array}{l}\text { Moyenne } \\
\text { Min.Max. }\end{array}$ & $\begin{array}{c}0,236 \\
0,22-0,26\end{array}$ & 8,0 & $\begin{array}{c}0,372 \\
0,36-0,39\end{array}$ & 9,1 & $\begin{array}{c}0,542 \\
0,48-0,58\end{array}$ & 12,0 & $\begin{array}{c}0,678 \\
0,58-0,70\end{array}$ & 13,6 \\
\hline Valérique & $\begin{array}{l}\text { Moyenne } \\
\text { Min,Max. }\end{array}$ & $\begin{array}{c}0,130 \\
0,11-0,14\end{array}$ & 4,4 & $\begin{array}{c}0,155 \\
0,15-0,17\end{array}$ & 3,8 & $\begin{array}{c}0,127 \\
0,12-0,14\end{array}$ & 2,8 & $\begin{array}{c}0,080 \\
0,07-0,09\end{array}$ & 1,6 \\
\hline Caproique & $\begin{array}{l}\text { Moyenne } \\
\text { Min.Max. }\end{array}$ & $\begin{array}{c}0,639 \\
0,58-0,65\end{array}$ & 21,7 & $\begin{array}{c}0,957 \\
0,94-0,97\end{array}$ & 23,4 & $\begin{array}{c}1,12 \\
1,08-1,22\end{array}$ & 24,8 & $\begin{array}{c}1,302 \\
1,24-1,33\end{array}$ & 26,1 \\
\hline Capryl ique & $\begin{array}{l}\text { Moyenne } \\
\text { Min.Max. }\end{array}$ & $\begin{array}{c}0,609 \\
0,58-0,61\end{array}$ & 20,7 & $\begin{array}{c}0,912 \\
0,85-0,93\end{array}$ & 22,3 & $\begin{array}{c}1,021 \\
0,94-1,13\end{array}$ & 22,6 & $\begin{array}{c}1,112 \\
1,09-1,24\end{array}$ & 22,5 \\
\hline $\begin{array}{l}\text { A.G.V.L. } \\
\text { totaux. }\end{array}$ & $\begin{array}{l}\text { Moyenne } \\
\text { Min.Max. }\end{array}$ & $\begin{array}{c}2,945 \\
2,69-3,09\end{array}$ & 100 & $\begin{array}{c}4,090 \\
3,85-4,12\end{array}$ & 100 & $\begin{array}{c}4,372 \\
4,37-4,64\end{array}$ & 100 & $\begin{array}{c}4,987 \\
4,79-5,13\end{array}$ & 100 \\
\hline
\end{tabular}


dégradés en acides gras à $\mathrm{n}-1$ atomes de carbone; la valine conduit ainsi à l'acide isobutyrique, la leucine à l'acide isovalérique et l'isoleucine à l'acide méthyl-3-butyrique (Harper, Kristoffersen et Wang, 1978). Les acides butyrique, caproïque et caprylique proviennent de la lipolyse, bien que le premier puisse être aussi d'origine fermentaire.

On peut remarquer que les teneurs en acides acétique, propionique, isobutyrique et valérique atteignent leurs valeurs maximales après le $1^{\text {er }}$ ou le $2^{\mathrm{e}}$ mois ; ensuite, elle diminuent. En revanche, les acides butyrique, caproïque et caprylique augmentent assez régulièrement pendant la maturation et ils dominent nettement le pool des A.G.L.V. en constituant près de $65 \%$ du total le $1^{\text {er }}$ mois et de $75 \%$ le $4^{\mathrm{c}}$ mois. Leur rapport, proche de $1 / 1 / 1$ (fig. 3), assure à la pâte un goût piquant agréable sans accuser de rancidité. On sait que l'un des caractères originaux de la matière grasse du lait de chèvre, que nous avons pu vérifier sur des échantillons de nos pays, est la présence des acides gras de 6 à 10 atomes de carbone en forte proportion relative, qui serait responsable de la flaveur caractéristique du lait de chèvre et du goût piquant de ses fromages. Selon Skjevdal (1979) cette flaveur est moins prononcée au début et en fin de lactation; elle présente de petites différences entre les périodes de pâturage et de stabulation; elle est généralement accentuée par une alimentaion intensive avec des aliments concentrés mais d'une façon générale elle est peu sensible aux facteurs alimentaires.

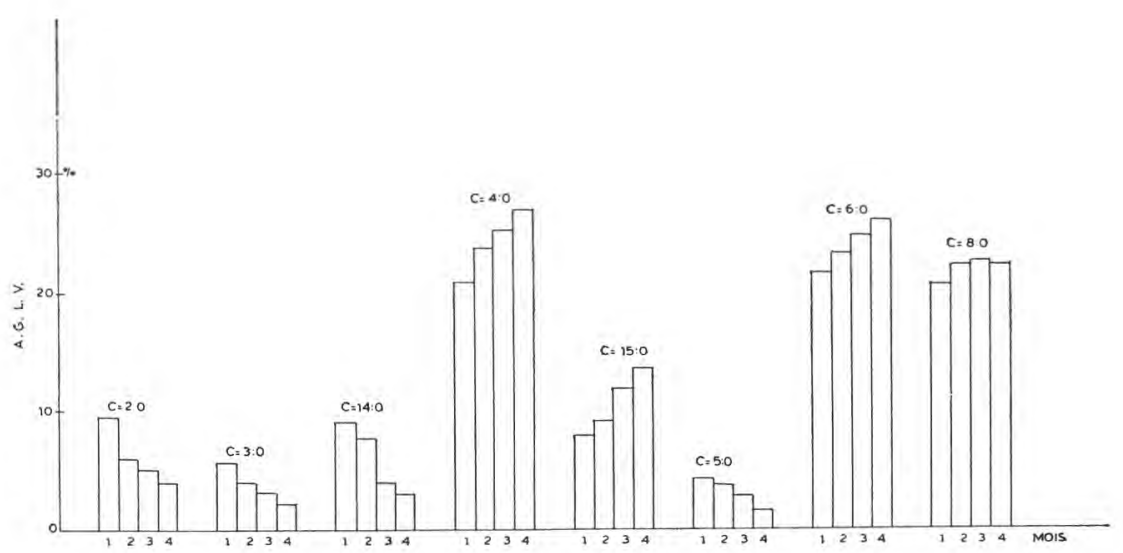

fig. 3

Variations de la composition en A.G.L.V. du fromage Rumelia au cours de l'affinage. (Proportions exprimées en pour cent des A.G.L.V. totaux).

Variations of volatile free fatty acids content in Rumelia cheese during ripening. (Proportions expressed in per cent of total volatile free fatty acids). 
Les analyses gustatives faites pendant l'affinage ont montré que les fromages étaient peu piquants après le $1^{\text {er }}$ mois mais trop après le 3e. En exprimant le taux d'A.G.L.V. provenant de la lipolyse par rapport à la matière grasse, les valeurs suivantes sont relevées aux temps 1 mois, $0,65 \%$; 2 mois, $0,95 \%$; 3 mois, $1,1 \%$; 4 mois, $1,25 \% \ldots$ Il se produit donc une importante libération d'acides gras volatils entre le $1^{\text {er }}$ et le $2^{\circ}$ mois.

La composition en A.G.L.V. des fromages fabriqués en Grèce était comparable à celle des fromages bulgares, avec toutefois une différence dans les teneurs en acides caproïque et caprylique, les fromages grecs étant plus riches en $6: 0$ et moins riches en $8: 0$, cette différence se retrouvant dans la composition en acides gras de la matière grasse des laits des deux origines (données non publiées).

Finalement, il est observé que les fromages Rumelia préparés en Grèce et en Bulgarie avaient, après 3 mois de maturation, une composition sensiblement identique et, grâce à leurs teneurs relativement élevées en acides aminés et en acides gras libres volatils un goût piquant, agréable et savoureux.

\section{B. Fromage type Edam}

1. Composition chimique, degré de protéolyse et composition en acides aminés

Nous avons cherché à préparer, selon la technologie du fromage Edam, une pâte semi-dure, souple, légèrement piquante, mais assez douce. Le produit final était homogène et sa composition chimique (tab. 5) correspond, selon les normes grecques, à une qualité " excellente ».

Malgré le délactosage du caillé, l'acidité a augmenté sensiblement pendant le $1^{\text {er }}$ mois, sans doute à cause de la faible teneur en sel.

L'évolution des diverses formes de l'azote au cours de l'affinage (tab. 6 et fig. 4) est voisine de celle observée sur le Rumelia; la composition en acides aminés libres (tab. 7) révèle cependant des quantités sensiblement plus faibles que dans ce dernier fromage.

La carte des acides aminés libres concrétisée par l'histogramme de la figure 5 , permet de relever là aussi une prédominance de l'acide glutamique et des proportions élevées de lysine et de leucine qui, ensemble en fin d'affinage, forment $45 \%$ des amino-acides libres totaux. Il est à remarquer que la libération des acides aminés est particulièrement active entre le $2^{\mathrm{e}}$ et le $3^{\mathrm{e}}$ mois d'affinage; ensuite elle est beaucoup moins forte mais les fromages sont alors conservés à $4^{\circ} \mathrm{C}$.

La comparaison des données des tableaux 3 et 7 permet d'observer certaines différences qui peuvent être dues à la nature 
TABLEAU $5-T A B L E 5$

Composition du fromage type Edam - Composition of Edam type cheese

\begin{tabular}{|c|c|c|c|c|c|}
\hline & 10 jours & 30 jours & 60 jours & 90 jours & 120 jours \\
\hline $\begin{array}{l}\text { Humidité \& * } \\
\text { (Min. - Max.) }\end{array}$ & $\begin{array}{c}44,7 \\
42,6-47,0\end{array}$ & $\begin{array}{c}42,7 \\
40,6-43,9\end{array}$ & $\begin{array}{c}41,7 \\
39,7-42,9\end{array}$ & $\begin{array}{c}39,2 \\
38,0-41,2\end{array}$ & $\begin{array}{c}38,7 \\
37,2-40,1\end{array}$ \\
\hline $\begin{array}{l}\text { Matière qrasse } 8 * \\
\quad(\text { Min.-Max, })\end{array}$ & $\begin{array}{c}25,4 \\
23,0 \cdot-27,4\end{array}$ & $\begin{array}{c}26,9 \\
24,5-28,1\end{array}$ & $\begin{array}{c}28,1 \\
26,7-29,4\end{array}$ & $\begin{array}{c}29,8 \\
27,3-31,2\end{array}$ & $\begin{array}{c}30,2 \\
28,6-32,1\end{array}$ \\
\hline $\begin{array}{l}\text { Matière grasse/ } \\
\text { Matière sèche\%* }\end{array}$ & 46,0 & 48,6 & 48,2 & 49,0 & 49,3 \\
\hline $\begin{array}{l}\text { Acidité en aci- } \\
\text { de lactique } z \text { * }\end{array}$ & 1,26 & 1,78 & 1,80 & 1,73 & 1,53 \\
\hline $\mathrm{NaCl} \&$ * & 0,1 & 1,75 & 2,45 & 2,68 & 2,85 \\
\hline
\end{tabular}

* Valeurs moyennes à divers temps de la maturation 
$T A B L E A U 6-T A B L E 6$

Evolution des fractions azotées du fromage type Edam au cours de la maturation Evolution in nitrogen constituent Edam type cheese of the during the ripening

\begin{tabular}{|c|c|c|c|c|c|}
\hline Fractions azotées* & 10 jours & 30 jours & 60 jours & 90 jours & 120 jours \\
\hline $\begin{array}{c}\text { Azote total (NT) } \\
\&\end{array}$ & 3,80 & 3,90 & 4,10 & 4,21 & 4,38 \\
\hline $\begin{array}{l}\text { Azote soluble } \\
\text { (\& NT) }\end{array}$ & 15,4 & 20,4 & 29,3 & 31,2 & 31,8 \\
\hline $\begin{array}{l}\text { Azote peptonique } \\
\left(\begin{array}{ll}8 & \text { NT }\end{array}\right)\end{array}$ & 12,9 & 14,9 & 20,3 & 20,8 & 20,4 \\
\hline $\begin{array}{l}\text { Azote aminé ** } \\
\left(\begin{array}{ll}8 & \text { NT }\end{array}\right)\end{array}$ & 2,5 & 5,5 & 9,0 & 10,4 & 11,4 \\
\hline $\begin{array}{l}\text { Azote aminé } * * \\
(8 \mathrm{~N} \text { Soluble) }\end{array}$ & 16,2 & 27,1 & 30,8 & 33,5 & 36,0 \\
\hline
\end{tabular}

* Valeurs moyennes à divers temps de la maturation

** Azote des acides aminés libres + azote ammoniacal 


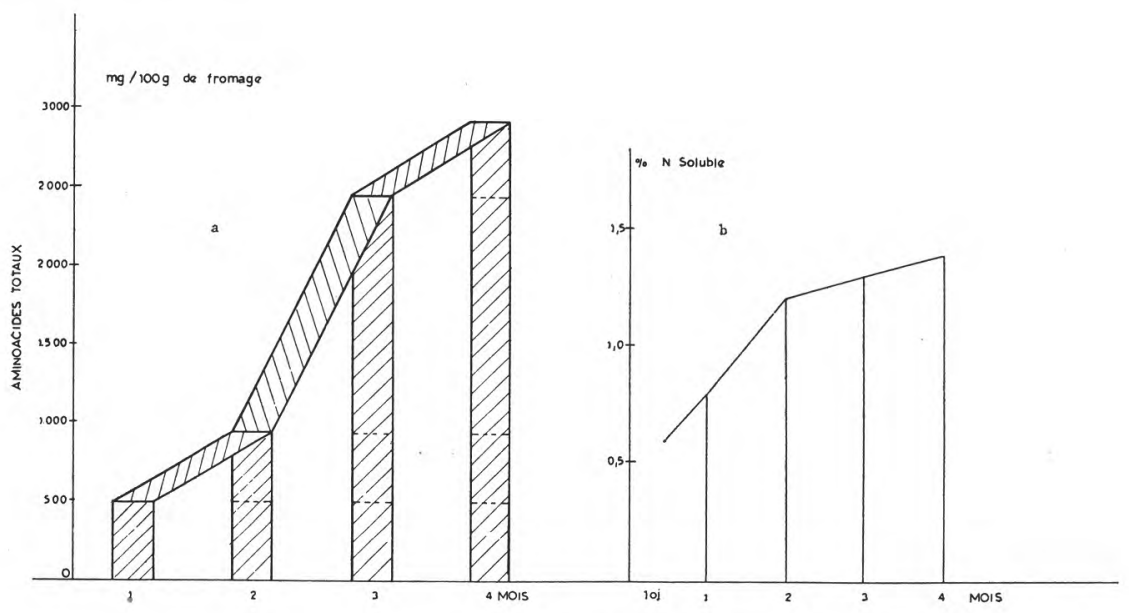

fig. 4

Evolution des teneurs en acides aminés libres (a) et en azote soluble (b) au cours de l'affinage du fromage type Edam.

Evolution of free aminoacids content (a) and of N.P.N. content (b) during ripening of Edam type cheese.

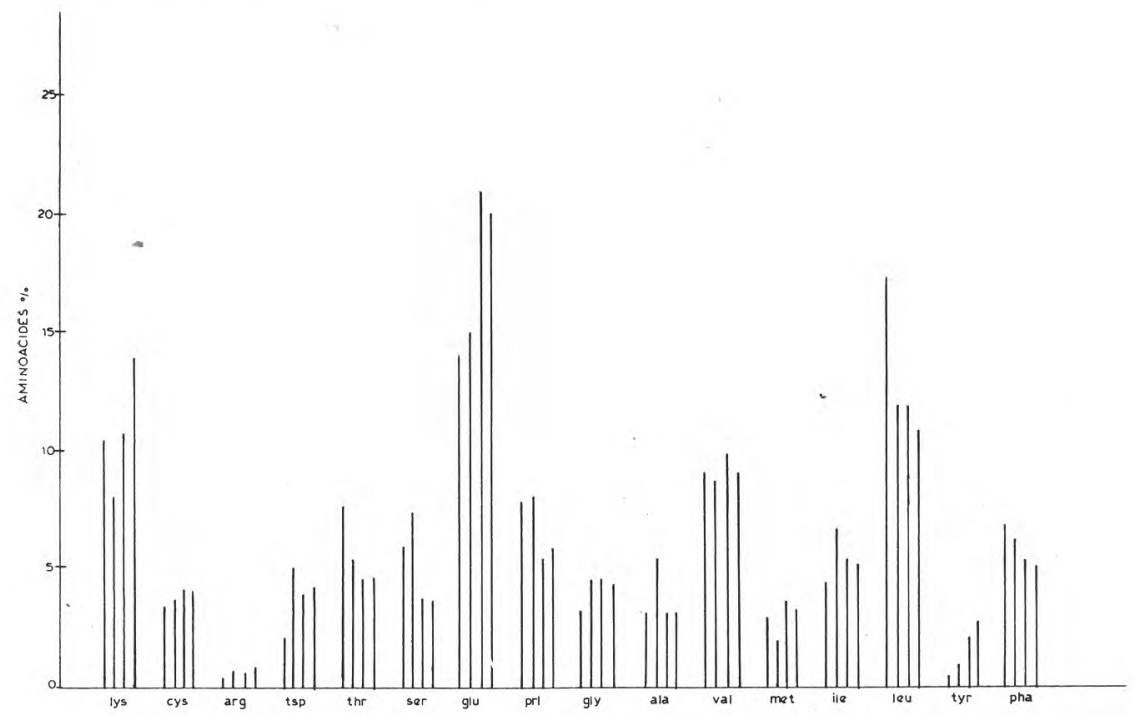

fig. 5

Variations de la composition en acides aminés libres du fromage type Edam au cours de l'affinage. (Proportions exprimées en pour cent des acides aminés totaux).

Variations of free aminoacids content of Edam type cheese during ripening. (Proportions expressed in per cent of total aminoacids). 


\section{TABLEAU 7 - TABLE 7}

Composition en acides aminés libres du fromage type Edam (en $\mathrm{mg} / 100 \mathrm{~g}$ de fromage)

Composition in aminoacids of Edam type cheese (in $\mathrm{mg} / 100 \mathrm{~g}$ of cheese)

\begin{tabular}{|c|c|c|c|c|c|c|c|c|c|c|c|}
\hline Aminoacides * & \multicolumn{2}{|c|}{1 mois } & $\%$ & \multicolumn{2}{|c|}{2 mois } & $\%$ & & mois & $\%$ & 4 mois & 8 \\
\hline Lysine & 51,00 & $\pm 2,98$ & 10,49 & 75,95 & $\pm 3,15$ & 8,06 & 266,98 & $\pm 12,16$ & 10,91 & $410,34 \pm 15,51$ & 14,03 \\
\hline Histidine & 16,42 & $\pm 1,31$ & 3,38 & 34,27 & $\pm 1,60$ & 3,64 & 103,19 & $\pm 3,52$ & 4,22 & $120,25 \pm 5,52$ & 4,11 \\
\hline Arginine & 1,26 & 0,20 & 0,26 & 5,59 & $\pm 0,99$ & 0,59 & 11,28 & $\pm 1,13$ & 0,46 & $21,65 \pm 0,65$ & 0,74 \\
\hline Aspartique (acide) & 9,94 & 1,05 & 2,04 & 46,37 & $\pm 1,98$ & 4,92 & 89,81 & 3,66 & 3,67 & $121,73 \pm 6,23$ & 4,16 \\
\hline Threonine & 39,27 & 1,99 & 8,08 & 50,15 & $\pm 2,29$ & 5,33 & 117,46 & 6,20 & 4,80 & $140,38 \pm 5,61$ & 4,80 \\
\hline Serine & 29,32 & 1,44 & 6,03 & 71,25 & $\pm 3,01$ & 7,57 & 94,73 & $\pm 8,91$ & 3,87 & $106,15 \pm 6,63$ & 3,63 \\
\hline Glutamique (acide) & 67,99 & 2,30 & 13,98 & 142,63 & $\pm 7,41$ & 15,15 & 520,16 & $\pm 25,53$ & 21,25 & $591,59 \pm 9,51$ & 20,23 \\
\hline Proline & 38,92 & 1,18 & 8,0 & 77,76 & $\pm 2,64$ & 8,26 & 130,80 & $\pm 5,89$ & 5,34 & $171,90 \pm 9,65$ & 5,88 \\
\hline Glycine & 15,59 & $\pm \quad 0,85$ & 3,21 & 41,10 & $\pm \quad 2,78$ & 4,36 & 108,60 & 4,00 & 4,44 & $124,47 \pm 5,08$ & 4,26 \\
\hline Alanine & 15,55 & $\pm 0,72$ & 3,20 & 51,37 & $\pm 3,04$ & 5,45 & 81,11 & 3,10 & 3,31 & $97,01 \pm 4,80$ & 3,32 \\
\hline Valine & 44,56 & $\pm 1,85$ & 9,16 & 81,96 & $\pm 2,59$ & 8,70 & 239,93 & $\pm 12,88$ & 9,80 & $265,43 \pm 16,74$ & 9,07 \\
\hline Methionine & 13,89 & $\pm 0,85$ & 2,86 & 17,86 & $\pm 2,00$ & 1,90 & 86,10 & $\pm 5,72$ & 3,52 & $93,68 \pm 3,69$ & 3,20 \\
\hline Isoleucine & 21,86 & 1,28 & 4,50 & 63,07 & $\pm 3,32$ & 6,70 & 130,76 & $\pm 7,14$ & 5,34 & $152,42 \pm 12,58$ & 5,21 \\
\hline Leucine & 84,86 & 4,15 & 17,45 & 113,43 & $\pm 6,66$ & 12,04 & 295,58 & $\pm 14,31$ & 12,07 & $328,12 \pm 14,10$ & 11,22 \\
\hline Tyrosine & 2,75 & $\pm 0,86$ & 0.57 & 10,30 & $\pm 1,30$ & 1,09 & 30,76 & $\pm 1,90$ & 1,26 & $38,55 \pm 2,79$ & 1,32 \\
\hline Phenylalanine & 33,58 & $\pm 1,31$ & 6,91 & 58,69 & $\pm 1,84$ & 6,23 & 132,54 & $\pm 5,76$ & 5,41 & $147,57 \pm 6,45$ & 5,05 \\
\hline Acides aminés $t$ & & 86,21 & 100 & & & 100 & & 48,04 & 100 & 2924,96 & 100 \\
\hline
\end{tabular}

* Valeurs moyennes à divers temps de la maturation. 
des levains lactiques utilisés. Avec les ferments mésophiles on note par exemple des proportions d'histidine et d'acide aspartique plus élevées, de sérine et d'isoleucine plus faibles qu'avec les levains thermophiles.

2. La composition en acides gras libres volatils

Les fromages examinés avaient un goût peu piquant, moins piquant que celui des fromages Rumelia; les teneurs en acides gras libres volatils sont d'ailleurs moindres (tab. 8) : 2,4 contre $2,9 \mathrm{~g} / \mathrm{kg}$ au $1^{\text {er }}$ mois, 4,6 contre $5,0 \mathrm{~g} / \mathrm{kg}$ au $4^{\mathrm{e}}$.

Les acides acétique, propionique, isobutyrique et valérique atteignent leurs valeurs maximales au $2^{\mathrm{c}}$ mois, ensuite leurs taux diminuent.

Les acides butyrique, caproïque et caprylique, qui sont les acides gras volatils dominants, augmentent pendant toute la durée de la maturation; ils constituent $60 \%$ des acides gras volatils totaux le $1^{\text {er }}$ mois et $85 \%$ le $4^{\mathrm{e}}$ (fig. 6). A ce stade l'acide caproïque, avec un pourcentage de $33 \%$, donne le goût caractéristique du fromage de chèvre, agréable aux consommateurs balkaniques.

La comparaison des données des tableaux 4 et 8 permet de relever des différences significatives dans le profil des acides gras libres volatils des deux types de fromages. Ces différences concernent notamment les proportions des acides isovalérique et valérique qui sont plus faibles dans le cas du fromage type Edam, alors que celles en acide acétique, caproïque et caprylique sont

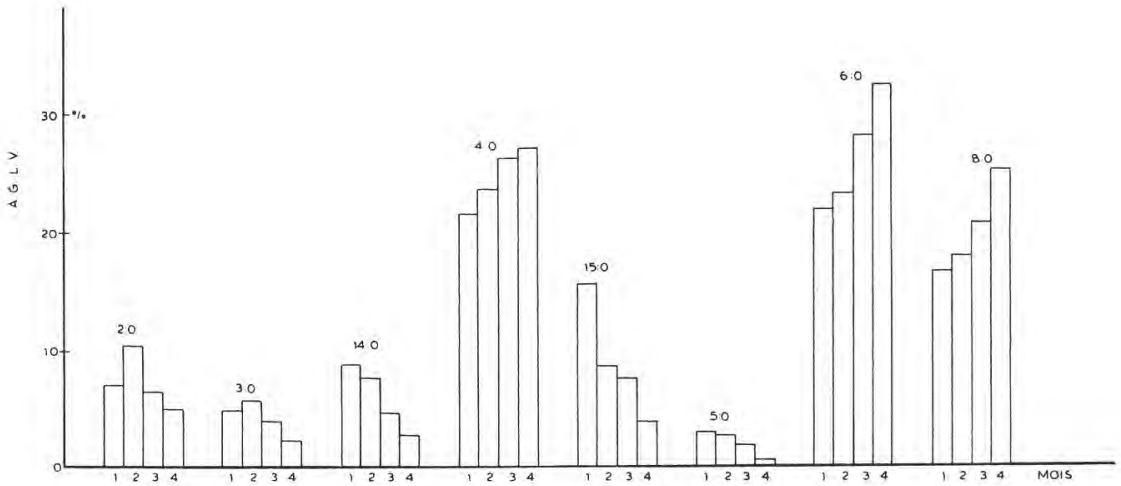

fig. 6

Variations de la composition en A.G.L.V. du fromage type Edam au cours de l'affinage. (Proportions exprimées en pour cent des A.G.L.V. totaux).

Variations of volatile free fatty acids content of Edam type cheese during ripening. (Proportions expressed in per cent of total volatile free fatty acids). 


$$
\text { TABLEAU 8-TABLE } 8
$$

Composition en acides gras libres volatils du fromage type Edam

Composition in volatile free fatty acids of Edam type cheese

\begin{tabular}{|c|c|c|c|c|c|c|c|c|c|}
\hline \multirow{2}{*}{\multicolumn{2}{|c|}{ Acides gras }} & \multicolumn{2}{|c|}{$1^{\text {er }}$ mois } & \multicolumn{2}{|c|}{$2^{\text {ëme mois }}$} & \multicolumn{2}{|c|}{$3^{\text {ëme mois }}$} & \multicolumn{2}{|c|}{4 ëme mois } \\
\hline & & $\mathrm{g} / \mathrm{Kg}$ From & $\%$ & $\mathrm{~g} / \mathrm{Kg}$ From & $\%$ & $\mathrm{~g} / \mathrm{Kg}$ From & $\%$ & $\mathrm{~g} / \mathrm{Kg}$ From. & $\%$ \\
\hline Acetique & $\begin{array}{l}\text { Moyenne } \\
\text { Min.Max. }\end{array}$ & $\begin{array}{c}0,176 \\
0,15-0,19\end{array}$ & 7,2 & $\begin{array}{c}0,345 \\
0,32-0,37\end{array}$ & 10,5 & $\begin{array}{c}0,257 \\
0,23-0,28\end{array}$ & 6,5 & $\begin{array}{c}0,219 \\
0,20-0,23\end{array}$ & 4,8 \\
\hline Propionique & $\begin{array}{l}\text { Moyenne } \\
\text { Min.Max. }\end{array}$ & $\begin{array}{c}0,122 \\
0,10-0,13\end{array}$ & 5,0 & $\begin{array}{c}0,189 \\
0,16-0,22\end{array}$ & 5,7 & 0,154 & 3,9 & $\begin{array}{c}0,105 \\
0,09-0,12\end{array}$ & 2,3 \\
\hline Isobutyrique & $\begin{array}{l}\text { Moyenne } \\
\text { Min.Max. }\end{array}$ & $\begin{array}{c}0,215 \\
0,20-0,23\end{array}$ & 8,8 & $\begin{array}{c}0,252 \\
0,22-0,28\end{array}$ & 7,6 & $\begin{array}{c}0,182 \\
0,17-0,20\end{array}$ & 4,6 & $\begin{array}{c}0,128 \\
0,11-0,31\end{array}$ & 2,8 \\
\hline Butyrique & $\begin{array}{l}\text { Moyenne } \\
\text { Min.Max. }\end{array}$ & $\begin{array}{c}0,527 \\
0,48-0,54\end{array}$ & 21,6 & $\begin{array}{c}0,789 \\
0,76-0,85\end{array}$ & 23,8 & $\begin{array}{c}1,046 \\
0,91-1,12\end{array}$ & 26,5 & $\begin{array}{c}1,252 \\
1,13-1,31\end{array}$ & 27,4 \\
\hline Isovalêrique & $\begin{array}{l}\text { Moyenne } \\
\text { Min.Max. }\end{array}$ & $\begin{array}{c}0,383 \\
0,35-0,41\end{array}$ & 15,7 & $\begin{array}{c}0,285 \\
0,26-0,30\end{array}$ & 8,6 & $\begin{array}{c}0,300 \\
0,28-0,31\end{array}$ & 7,6 & $\begin{array}{c}0,187 \\
0,17-0,19\end{array}$ & 4,1 \\
\hline Valêrique & $\begin{array}{l}\text { Moyenne } \\
\text { Min.Max. }\end{array}$ & $\begin{array}{c}0,078 \\
0,06-0,09\end{array}$ & 3,2 & $\begin{array}{c}0,089 \\
0,08-0,09\end{array}$ & 2,7 & $\begin{array}{c}0,075 \\
0,07-0,08\end{array}$ & 1,9 & $\begin{array}{c}0,037 \\
0,03-0,04\end{array}$ & 0,8 \\
\hline Caproique & $\begin{array}{l}\text { Moyenne } \\
\text { Min.Max. }\end{array}$ & $\begin{array}{c}0,536 \\
0,45-0,60\end{array}$ & 22,0 & $\begin{array}{c}0,772 \\
0,75-0,80\end{array}$ & 23,3 & $\begin{array}{c}1,113 \\
1,01-1,24\end{array}$ & 28,2 & $\begin{array}{c}1,489 \\
1,28-1,56\end{array}$ & 32,6 \\
\hline Caprylique & $\begin{array}{l}\text { Moyenne } \\
\text { Min.Max. }\end{array}$ & $\begin{array}{c}0,402 \\
0,38-0,43\end{array}$ & 16,5 & $\begin{array}{c}0,593 \\
0,54-0,63\end{array}$ & 17,9 & $\begin{array}{c}0,821 \\
0,74-0,89\end{array}$ & 20,8 & $\begin{array}{c}1,151 \\
1,06-1,29\end{array}$ & 25,2 \\
\hline $\begin{array}{l}\text { A.G.L.V. } \\
\text { TOTAUX }\end{array}$ & $\begin{array}{l}\text { Moyenne } \\
\text { Min.Max. }\end{array}$ & $\begin{array}{c}2,439 \\
2,26-2,67\end{array}$ & 100 & $\begin{array}{c}3,315 \\
3,12-3,58\end{array}$ & 100 & $\begin{array}{c}3,949 \\
3,75-4,12\end{array}$ & 100 & $\begin{array}{c}4,569 \\
4,36-4,79\end{array}$ & 100 \\
\hline
\end{tabular}


plus élevées. Grâce à ces différences le goût et l'arôme des deux fromages étaient bien distincts.

Parallèlement, des fromages préparés en Grèce selon la même technologie ont été examinés. Certaines différences statistiquement significatives ont été observées dans la composition en acides aminés et dans celle en A.G.L.V.

Au point de vue gustatif les fromages grecs étaient de meilleure qualité, plus savoureux et plus doux.

Comme il a été dit précédemment un autre groupe de nos laboratoires a mis au point et étudié le fromage Fildrus, dont la

\section{TABLEAU $9-T A B L E 9$}

Composition en acides aminés libres du fromage semi-dur type Edam et du fromage Fildrus

Composition in free aminoacids of Edam type cheese and Fildrus cheese

\begin{tabular}{|c|c|c|c|}
\hline Acides aminés* & $\begin{array}{c}\text { Semi-dur } \\
(1)\end{array}$ & $\begin{array}{c}\text { Fildrus } \\
(2)\end{array}$ & $\begin{array}{c}\text { Différence } \\
(1,-(?)\end{array}$ \\
\hline Lys & $267,00 \pm 12,16$ & $285,35 \pm 14,64$ & $-18,35$ \\
His & $103,20 \pm 3,52$ & $28,60 \pm 3,61$ & $+74,60$ \\
Arg & $11,28 \pm 1,13$ & $7,03 \pm 1,44$ & $+4,25$ \\
Aso & $89,81 \pm 3,70$ & $69,43 \pm 7,22$ & $+20,38$ \\
Thr & $117,46 \pm 6,2$ & $35,61 \pm 3,42$ & $+81,85$ \\
Ser & $94,73 \pm 8,91$ & $117,44 \pm 10,63$ & $-22,71$ \\
Glu & $520,16 \pm 25,53$ & $330,99 \pm 24,35$ & $+189,17$ \\
Pro & $130,80 \pm 5,89$ & $151,93 \pm 9,74$ & $+21,13$ \\
Gly & $108,60 \pm 4,00$ & $88,40 \pm 8,94$ & $+20,20$ \\
Ala & $81,11 \pm 3,10$ & $95,40 \pm 7,27$ & $-14,29$ \\
Val & $239,90 \pm 12,88$ & $265,35 \pm 20,35$ & $-25,45$ \\
Met & $86,01 \pm 5,72$ & $87,96 \pm 80,73$ & $-1,95$ \\
Ile & $130,76 \pm 7,14$ & $166,96 \pm 10,33$ & $-36,20$ \\
Leu & $295,58 \pm 14,31$ & $237,45 \pm 14,27$ & $+58,13$ \\
Tyr & $30,76 \pm 1,90$ & $4,64 \pm 1,30$ & $+26,12$ \\
Phe & $132,54 \pm 5,76$ & $155,67 \pm 8,34$ & $-23,13$ \\
ToTAL & 2448,04 & 2442,07 & \\
& & & \\
\hline
\end{tabular}

* Vaieurs moyennes de sept préparations. 
technologie est voisine de celle utilisée pour le fromage semi-dur, la distinction tenant essentiellement à la nature des ferments utilisés qui, pour le Fildrus, sont des bactéries lactiques thermophiles, Streptocaccus thermophilus et Lactobacillus bulgaricus (Baltadjieva et al., 1978; Baltadjieva, Stamenova et Anifantakis, 1983). Le tableau 9 met en évidence les différences dans la composition en acides aminés libres des deux types de fromages après 3 mois d'affinage. On peut constater que pour un taux global d'acides aminés libres comparable, les proportions relatives de certains d'entre eux sont très différentes, par exemple l'histidine, la thréomine, l'acide glutamique, la tyrosine sont beaucoup plus abondants dans le fromage semi-dur alors que la sérine, l'isoleucine ou la phénylalanine y sont présents en moindre quantité.

\section{v. CONCLUSION}

Cette étude a permis de caractériser l'évolution, au cours de l'affinage, de la composition en différentes fractions azotées, en acides aminés et en acides gras libres volatils de deux nouveaux types de fromages préparés à partir de lait de chèvre.

Le fromage Rumelia, après 2 mois d'affinage, présente un coefficient de maturation élevé (32\%), mais sa teneur en acides aminés libres est encore faible $(1,14 \mathrm{~g} / \mathrm{kg})$ comparée à celle atteinte après 3 mois $(3,16 \mathrm{~g} / \mathrm{kg})$.

Parmi les acides gras libres volatils trouvés en quantités importantes dès le $2^{c}$ mois, les acides caprö̈que et caprylique, caractéristiques de la matière grasse du lait de chèvre, donnent au fromage le goût piquant recherché.

Le fromage type Edam, malgré le délactosage du caillé, présente après 2 mois d'affinage un coefficient de maturation (29\%) et une teneur en acides aminés $(0,94 \mathrm{~g} / \mathrm{kg})$ proches de ceux du fromage précédent; les différences de composition en acides aminés libres observées entre les deux types de fromage sont également faibles; elles traduisent le fait que les actions de dégradation secondaire des amino-acides sont dans l'un et l'autre cas plutôt discrètes.

Les teneurs en A.G.L.V. ne sont pas non plus très différentes; toutefois, les profils présentent certaines caractéristiques typiques, on relève notamment des proportions d'acide isovalérique particulières à chacune des pâtes.

\section{REFERENCES BIBLIOGRAPHIQUES}

Aston J.W., Dulley J.R., 1982. Cheddar cheese flavour. Aust. J. Dairy Technol., 37, 59-63. 
Baltadjieva M., Stamenova V., Veinoglou B., Anifantakis E., 1978. Technology for the production of hard cheese from goat milk. Brevet du Gouvernement Bulgare, $\mathrm{n}^{\circ} 40880 / 78$.

Baltadjieva M., Stamenova V., Anifantakis E., 1983. Studies on the amount of free aminoacids in the Fildrus cheese. Travaux scientifiques de l'Institut Technologique supérieur des industries alimentaires, Plovdiv, Tome XX, X, C.B.I., $1-8$.

Baltadjieva M., Veinoglou B., Kalatzopoulos G., Stamenova V., Sfakianos A., 1984. La préparation d'un nouveau type de fromage "Rumelia " fait à partir du Iait de chèvre. Sci. Tec. Latt. Casearia (sous presse).

Choisy C., Desmazeaud M., Gripon J.C., Lamberet G., Lenoir J, Tourneur Colette, 1984. Les phénomènes microbiologiques et enzymatiques et la biochimie de l'affinage, in Le Fromage, Eck A., p. 62-100, Technique et Documentation, Paris.

De Vuyst A., Vervack, W., Vanbelle M., Foulon M., 1973. La composition en acides aminés de quelques fromages courants. Lait, 53, 625-632.

Dimitrov D., Obretenova M., Obretenov T., 1976. Etude sur l'arôme de Kaskavale. Travaux scientifiques de l'Institut supérieur des industries alimentaires, Plovdiv, I, XX, III, part II.

Do Ngoc M., Lenoir J., Choisy C., 1971. Les acides aminés libres des fromages affinés de Camembert, Saint-Paulin et Gruyère de Comté. Rev. Lait. Fr., (288), 447-459.

HaRPER W., KRISTOFFerSEn T., Wang J., 1978. Formation of free fatty acids during the ripening of fat modified cheese slurries. Milchwissenschaft, 33, 604-608.

INIHOF G.C., BRIo N.P., 1971. Méthodes d'analyse du lait et des produits laitiers. Moscou.

Kalatzopoulos G., Veinoglou B., Baltadjieva M., Alexandropollos C., Stamenova V., Sfakianos A., 1983. Préparation d'un fromage semi-dur fait à partir du lait de chèvre. Lait, 63, 345-361.

Kaspryk P., Michel J.F., Seure A.M., Mathalouthi M., 1983. Maturation des fromages à pâte pressée cuite de type Emmental. Ed. Apria.

Kuzdzal-Savoie S., Kuzdzal W., Coto K., Trehin J., 1971. Les acides gras libres du Munster. Rev. Lait. Fr., (290), 615-629.

LAW B.A., 1981. The formation of aroma and flavour compounds in fermented dairy products. Dairy Sci. Abstr., 43, 143-154.

Lenotr J., 1962. Sur la dégradation des protides au cours de la maturation du Camembert. C.R. Acad. Agric., 48, 160-169.

LeNOIR J., 1963. Note sur la composition en matières azotées des fromages affinés de Camembert, Saint-Paulin et Gruyère de Comté. Ann. Technol. Agric., 12, 51-57.

Polychroniadou A., Vlachos J., 1979. Les acides aminés du fromage Télémé, Lait, $59,234-243$.

RASic J., Vucurovic N., OBRadovic B., 1973. - Untersuchung der Fettsäurenzusammensetzung in Joghurt aus Kuh-, Schaf- und Ziegenmilch. Milchwissenschaft, 28, 168-171.

SKJEVDAL T., 1979. Flavour of goat's milk. A review of studies on the sources of its variations. Lives. Prod. Sci., 6, 397-405.

Veinoglou B., Baltadjeva M., Kalatzopoulos G., Dalles T., Stamenova V., 1983. Préparation des fromages frais et à pâte molle faits à partir du lait de chèvre. Rev. des Etudes agricoles, Athènes, Ed. Bank Agricole de Grèce, 4, sept,. 16-29. 